\title{
Germanica
}

\section{Amanda herzlos Rückblicke auf die DDR in der erzählenden Prosa seit 1991}

Amanda herzlos. Regards sur la RDA dans la prose narrative depuis 1991

Lothar Köhn

\section{OpenEdition}

1 Journals

Édition électronique

URL : http://journals.openedition.org/germanica/2168

DOI : $10.4000 /$ germanica. 2168

ISSN : 2107-0784

Éditeur

Université de Lille

\section{Édition imprimée}

Date de publication : 31 décembre 1993

Pagination : 41-61

ISSN : 0984-2632

\section{Référence électronique}

Lothar Köhn, « Amanda herzlos Rückblicke auf die DDR in der erzählenden Prosa seit 1991 », Germanica [Online], 13 | 1993, Online erschienen am: 07 Februar 2014, abgerufen am 06 Oktober 2020. URL : http://journals.openedition.org/germanica/2168 ; DOI : https://doi.org/10.4000/germanica.2168

Ce document a été généré automatiquement le 6 octobre 2020.

(c) Tous droits réservés 


\title{
Amanda herzlos Rückblicke auf die DDR in der erzählenden Prosa seit 1991
}

Amanda herzlos. Regards sur la RDA dans la prose narrative depuis 1991

\author{
Lothar Köhn
}

1 Die Ich-Erzählung hat Konjunktur, aber seit 1990 haben Erzählungen einiger Autoren im Kontext der deutschen Vereinigung eine in den Massenmedien verhandelte öffentliche Brisanz gewonnen, von der Wolfgang Koeppen (Jugend, 1976) oder Peter Handke, um bloß diese Ich-Erzähler zu nennen, nur träumen konnten. Schon der sogenannte Literaturstreit um Christa Wolfs Was bleibt $(1990)^{1}$ ließ z.T. erstaunliche Prämissen erkennen. Das literarische Ich des Textes wurde ungeniert autobiographisch verstanden, als liefere da jemand eine gerichtsnotorische Aussage ab. Als sei die Literarisierung des Autobiographischen nie beschrieben worden, als sei, allgemeiner gesagt, das «Ich», das sich da (fingiert oder «authentisch») artikuliert, gerade in der erzählenden Prosa der Moderne niemals als «unrettbar» (H. Bahr) erkannt worden, als habe es niemals Diskussionen über den Zusammenhang von Sinnprojektion, IchIdentität und Erzählform gegeben, als hätten nicht seit den Zwanziger Jahren Debatten über die Legitimität «biographischen» Erzählens stattgefunden, um nur ein paar Binsenweisheiten ins Gedächtnis zu rufen². Damit soll der referentielle Inhalt des Erzählens nicht prinzipiell in Frage gestellt, das Autobiographische nicht a priori der allgegenwärtigen Fiktion anheimgegeben werden. Vielmehr ist der Fiktionalisierungsgrad eines Textes je konkret zu bestimmen und zu beachten.

2 Man darf im übrigen weiterhin unterstellen, daß es den Fiktionen aufgegeben ist, Wahrheit zur Sprache zu bringen, wo die Dokumente ganz offenbar versagen oder doch nur bestätigen, was Literarhistoriker seit langem als Einmaleins moderner Realitätserfahrung wiederholen, nämlich daß diese immer perspektivisch verzerrt sei was gerade die Fiktion thematisch macht. In diesem Zusammenhang ist ein Argument der jüngsten Literaturdebatte von Bedeutung. «Die deutsche Gesinnungsästhetik», so hatte man verkündet, sei jetzt zu Ende und mit ihr eine Epoche der neuesten deutschen 
Literatur. «In der Gesinnungsästhetik [...] sind Werk und Person und Moral untrennbar. Der Text ist der moralische Selbstentwurf des Autors. Und der Autor ist identisch mit seiner moralischen Absicht. Diese Moral beruft sich auf Humanität und Universalität. [...] So einfach ist das» ${ }^{3}$. So einfach ist es keineswegs. Es ist aber auch nicht so, als habe nun die Moral in einen Lebenszusammenhang abzuwandern, dessen literarische Zeugnisse beispielsweise Aktenberge und autobiographische Bekenntnisse wären. Damit würden sich allerdings die moralischen Sachverhalte, von der Bürde ästhetischfiktionaler Komplexität befreit, so vereinfachen, daß jeder Feuilletonist, und nicht nur der, erfolgversprechend mitreden könnte. Man kann beispielsweise darüber diskutieren, wie moralisch es sei, daß Christa Wolf einmal IM Margarete war $^{4}$, und ob das durch ihre und ihres Mannes Akten aufgewogen würde, die sie als operativen Vorgang «Doppelzüngler» führten; ob Heiner Müller seine Anmerkungen zu StasiKontakten (IM Heiner) nicht doch in sein autobiographisches Buch Krieg ohne Schlacht hätte aufnehmen sollen ${ }^{5}$, von Kants IM Martin ganz zu schweigen, der natürlich in seinem Abspann nicht auftaucht ${ }^{6}$. Das bedeutet, daß man der alltäglichen Lebenserfahrung eine klare Moral unterstellt (und das Recht dazu soll gar nicht bestritten werden, obwohl die angeblich universale Moral oft nur die des Argumentierenden ist). Demgegenüber sind die Binsenweisheiten, von denen zuvor die Rede war, also die Charakteristika ästhetischer Fiktionalisierung, allerdings als Erkenntnispotentiale auch (literarisch-) moralischen Zuschnitts zu behaupten, für die es gerade gegenwärtig kein Äquivalent geben kann. Das zeigt sich beispielsweise, wenn Erkenntnisse der modernen Literatur sich unvermutet als Lebenswahrheit erweisen, wie es Günter de Bruyn unter der Überschrift «Dies Mißtrauen gegen mich selbst. Schwierigkeiten beim Schreiben der Wahrheit» mit Brecht- und ChristaWolfAnspielungen belegte, als er von seiner Krise angesichts seiner Stasi-Akten berichtete, die ihn als IM Roman führten ${ }^{7}$. Nicht die Kategorien einer (auch möglichen) ästhetischen Spielwelt, sondern Kategorien «moralischer» Literatur, sofern sie keine ideologische Erbauungsliteratur ist, zeigen sich als lebensbedeutsam. Ließe sich die literarische Wahrnehmungsform der Mutmaßung nicht auf die deutschen Verhältnisse übertragen und als angemessen erkennen, hätte Uwe Johnson mit Mutmaßungen über Jakob (1959) und mit seinem Dritten Buch über Achim (1961) nicht Maßstäbe setzen können, die auch vor dem aktuellen Hintergrund dokumentierbarer Sachverhalte ihre prinzipielle Bedeutung kaum verlieren können.

3 «Rückblicke auf die DDR» ist unter solchen Voraussetzungen nicht in erster Linie ein «inhaltliches» Thema, das von den Texten dokumentarische Auskunft über die Korrumpierbarkeit ihrer Verfasser, der literarischen Intellektuellen verlangt. Es soll vielmehr daran erinnert werden, daß Erzählprosa einen genuinen Zugriff auf Wirklichkeit besitzt, der die alltägliche Mischung aus Urteilen und Vorurteilen sehr wohl korrigieren kann. Der neue Roman von Jurek Becker bildet deshalb den Fokus meiner Überlegungen, weil er die thematische Struktur, um die es hier geht, exemplarisch aufzeigt, und das gerade, weil Amanda herzlos ein eindeutig fiktionaler Text mit drei fingierten Ich-Erzählern ist. Zudem ist in der Widersprüchlichkeit des Titels einiges von der Widersprüchlichkeit der alten DDR-Thematik ausgesagt, sicher eher ironisch als allegorisch, denn nicht die «zu Liebende» allein repräsentiert in dem Roman die DDR. Amanda herzlos bezeichnet also den Pol der Fiktionalisierung der IchErzählung. Den entgegengesetzten Pol der neuen Ich-Prosa markieren die eindeutig autobiographischen Texte, beispielsweise Hermann Kants Abspann. Erinnerung an meine Gegenwart (1991) oder Heiner Müllers Krieg ohne Schlacht. Leben in zwei Diktaturen (1992), 
aus einem großen Interview erarbeitet, aber keinesfalls literarisch zweite Wahl. Das Ich-im-Text trägt hier den Namen der Autoren, die Wirklichkeit saussagen sollten im Prinzip überprüfbar sein. Daß richtiges Leben im falschen - um Adorno zu paraphrasieren - nicht möglich gewesen sein kann, bestätigen beide Autobiographien nicht, natürlich auf sehr unterschiedliche Weise ${ }^{8}$.

Bei Hermann Kant, bis 1989 Präsident des Schriftstellerverbandes der DDR, ist das nicht verwunderlich. Aber schon ein erster Blick in sein Buch zeigt, daß Sachverhalte, die scheinbar einfach waren, sich durch das Erzählverfahren komplizieren. Man könnte kritisch einwenden, daß die Literarisierung, die Vor- und Rückgriffe, Ironie, Invektiven, Vermischung von Fiktion und Realität bei ihm der Verschleierung dienen, aber so einfach ist das selbst hier nicht. Ich gebe nur ein Beispiel; es gehört in den Zusammenhang einer Lesung Kants in Bad Godesberg im Oktober 1969:

Mich weiter zu entspannen, schickten Smileys Leute einen Kerl gegen mich vor, der sich benahm, als habe Erich Loest ihn ersonnen. Er trug tatsächlich einen ehedem cremefarbenen schmuddeligen Ledermantel und versuchte tatsächlich, mir fünfzig Westmark in die bücher signier ende Hand zu drücken [...] (K 306) ${ }^{9}$.

Die weiteren «tatsächlich» brauche ich nicht zu zitieren. Die Markierung eines Vorgangs als «Tatsache», die überprüfbar sein müßte, soll diese westliche Wirklichkeit als schlechte Literatur ausweisen, die auf ihre Verfasser, insbesondere auf Loest zurückfällt, der seinerseits als mieser DDR-Realist erscheint. Obwohl er damals seine Kriminalromane, so kann der Kommentator ergänzen, in der DDR unter Pseudonym schreiben und sich alles Realistische ausdenken mußte, weil er nach seiner Entlassung aus der Haft offiziell Publikationsverbot hatte und im Gegensatz zum Reisekader Kant niemals im Westen gewesen war. So etwa ließe sich Kants Literarisierung der Tatsachen kritisch auf ihren sachlichen Boden zurückführen, auf den es ihm ja ankommt. Kants assoziativer Humor verwischt die Konturen nur oberflächlich, in Wirklichkeit zeigt er ein unbewegt-abgedichtetes, seiner selbstscheinbar sicheres apologetisches Ich. «Ich habe vieles unterlassen, weil ich fürchtete, es werde der anderen Seite dienen - wozu also, fragt sich heute, habe ich es unterlassen?» (K 531) Die Weltordnung mit der «anderen Seite» wurde dem Ich bloß entzogen, und das passiv-unpersönliche «fragt sich» anstelle eines «frage ich» ist nicht bloß ein Stilistikum, wenn es kurz zuvor vielversprechend hieß: «Zuallererst habe ich mich an mich selbst zu halten» (K 530).

6 Das erzählende Ich Kants muß apologetisch bleiben, weil nur so seine Scheinidentität auf dem Boden scheinbarer Tatsachen gewahrt bleiben kann. Ganz anders Heiner Müllers Buch. Daß es aus Interviews mit drei Gesprächspartnern im Lauf von Monaten entstanden ist, entnimmt man nur dem Waschzettel; daß das Manuskript ursprünglich viel umfangreicher war, hat Müller in begleitenden Äußerungen mitgeteilt. Das schließlich vorgelegte Buch fingiert ein an der biographischen Chronologie orientiertes Interview, dessen Fragesteller anonym bleibt; die Spontaneität des Mündlichen scheint gewahrt, aber sie ist partiell «gestellt». «Die Kunst des Erzählens in den Medien ist verloren gegangen, auch mir seit dem Verschwinden des Erzählers in den Medien, der Erzählung in der Schrift» (M 366) ${ }^{10}$. Dieser Selbstkommentar auf der vorletzten Seite trifft genau die Form des Textes, ist doch das Interview auch eine der schlechthin medialen Formen. Das Printmedium überführt das mündliche Erzählen in die Schrift. Der Autor-Bearbeiter konzentriert sie zur Literatur, nicht etwa, um die umfangreichere Vorlage kohärent $\mathrm{zu}$ machen. «Deshalb der vorliegende disparate Text, der problematisch bleibt» (366). Das sprechende Ich veröffentlicht sich in seiner Rolle als 
Schriftsteller, dem alles, es selbst, die Tatsachen, die Politik, zum literarischen Material geworden ist.

Die DDR, die ich, im Doppelsinn des Wortes, beschrieben habe, die Beschreibung ist auch eine Übermalung, war ein Traum, den Geschichte zum Alptraum gemacht hat, wie das Preußen Kleists und Shakespeares England (363).

7 Die moderne Kunstform der Übermalung als Schrift über einem Bild, also keine «Bildbeschreibung». "Was "Bildbeschreibung" angeht: Das kann jeder machen», so Müller kurz zuvor über seinen rätselhaften Kurzprosa-Text, aber er fügt hinzu «die Beschreibung produziert neue Bilder, wenn er mitschreibt, was ihm einfällt während der Beschreibung» (343). Neue Bilder als Übermalungen, die als Schrift zu sekundären Beschreibungen werden. Das gilt auch für die eigene Biographie. Dazu stimmen die Metaphern Traum / Alptraum für die DDR, stimmt die Analogie zwischen Ich, Kleist und Shakespeare. Nur ein erratischer Block, «Geschichte», bleibt als geradezu mythischer Agent im Relativsatz stehen. Aber der Eindruck muß genügen, um das autobiographische Ich in seiner medialen Kunstform vorzustellen.

Eine Übermalung ganz anderer Art hat Erwin Strittmatter mit dem dritten Band seiner Trilogie Der Laden ${ }^{11}$ gegeben. Er soll nicht nur als Beispiel für den Übergang zum autobiographischen Roman genannt werden, sondern vor allem, weil dieser Roman ein Bild der Realität entwirft, das sich von Müllers Literarisierung, aber auch von der Tatsachen-Apologetik Kants unterscheidet. Der Laden, schon durch Esau Matt, den Namen des IchErzählers, deutlich fingier - wie bei Strittmatters vorausgehenden Parallelunternehmungen - ist des Autors dritter Versuch, den Lebenslauf eines kleinen Helden zwischen Erstem Weltkrieg und dem ersten Jahrzehnt der DDR zu erzählen. Dem Laden waren Ochsenkutscher (1950) und drei Bände Wundertäter vorausgegangen, Lebenslauf-Variationen mit immer stark autobiographischen Anteilen. Jetzt also der «Roman Dritter Teil» der neuen Trilogie (Bd. 1 1983, Bd. 2 1987). Bemerkenswert ist die Rezeptionsgeschichte dieses dritten Teils im Jahre 1992: Vor vollen Sälen las Strittmatter in Ostdeutschland aus seinem Roman, der binnen kurzem eine Auflage von 50.000 Exemplaren erreichte. Sie wurden offenbar überwiegend in den neuen Bundesländern verkauft; im Westen konnte Strittmatter, wie er selbst beklagte, «die Mauer» nicht durchbrechen ${ }^{12}$. Wie läßt sich diese Wirkung im Osten erklären? Strittmatter hat, gelegentlich literaturpolitisch behelligt, aber auch Schulautor, immer in der DDR gelebt, ohne sich zu exponieren wie Kant. Das und sein ehrwürdiges Alter (Jahrgang 1917) mögen eine Rolle spielen. Wichtiger ist sein Roman selbst. Wenn viele der neuesten (autobiographischen) Ich-Erzählungen im Rückblick auf die DDR zwischen den Extremen des Apologetischen und der totalen Kritik (also den alten Polen) ${ }^{13}$ angesiedelt sind, so besetzt Strittmatters Laden den Ort der gleichmütigen Selbstvergewisserung von Lebenserfahrung. Er versucht deren Rettung durch eine fingiert naive Erzählhaltung, die nichts zu verwerfen braucht, weil schlechthin alles, was erlebt wurde, auch das Widerständige, sinnvoll dazu gehört. Das Alltäglichste dörflicher Kleinbürger-Idylle - von der kritischen Heimatliteratur ist Strittmatter ein gutes Stück entfernt - wird im Präsens sozusagen hautnah und detailversessen, den Gestus mündlichen Sprechens wahrend, herunter erzählt. Das Politische ist (ähnlich wie bei Johnson oder Bobrowski) unüberhörbar, aber episodenhaft eingefügt. So liest man zu den Verhaftungen durch die Sowjets in der frühen Nachkriegszeit: «Ists möglich, daß sich nach kurzer Zeit Gleiches unter anderen Vorzeichen wiederholt und daß wieder geschieht, was soeben noch mit Abscheu bedacht wurde?» (S 415). Sogleich aber setzt der präsentische Strom der Details wieder ein, ironisch beleuchtet, ja 
weltweise kommentiert: «Ihr habt es alle schon erlebt, daß etwas Schlimmes, was ihr befürchtet, sich über Nacht in etwas Angenehmes verwandelte» (417). Esau, unter Hitler als Sozialdemokrat belangt, ist wieder verdächtig, aber das alles wird eingebettet in die Suada seines Erzählens und wohlwollendresignativen Besprechens, in der erlebendes und erzählendes Ich nicht zerspalten, sondern eins sind. «Glaubt es oder glaubt es nicht: Das Neue ist das Alte, das nur seine Form ein wenig veränderte, damit man es nicht gleich erkennt» (462). Die gerettete Biographie als Zuspruch, das mehrschichtige Semper idem hinzunehmen. Nicht kritiklos zwar, doch müßte jede Verabsolutierung der kritischen, gar selbstkritischen Haltung zum totalen Sinnverlust dieser Fiktion führen. Wo Kant apologetisch sein «Ich» stärkt, Müller auf Literatur reflektiert, verweist Esau Matts Erzählhaltung auf das «Wir» der Phänomene, der fingierten Biographie und des Leser-Alltags.

9 «Mein Interesse an mir ist am heftigsten, wenn ich über andre rede» (M 366), schreibt Heiner Müller. Die fingierte IchErzählung kann das beispielsweise realisieren, indem sie die Ich Erzählerin in den Vordergrund rückt, sie aber gleichzeitig zur Biographin eines Gegenbildes macht. So verfährt Monika Marons Roman Stille Zeile sechs (1991) ${ }^{14}$. Wenn Strittmatters Laden ein großes Identifikations -, ja ein Trostangebot für Durchschnittsleser bereitstellt, die all das miterlebt haben, was Esau Matt ihnen wiedererzählt, verweigert Marons Roman bewußt jede Heimeligkeit, aber letztlich auch die platte Opposition des Guten und Bösen, die der Aufbau ihres Romans nahelegt. Während nämlich die Ich-Erzählerin der informellen Opposition in der DDR nahesteht, begegnet ihr in dem Altkommunisten Prof. Beerenbaum, der ihr seine Autobiographie diktiert, eine greisenhafte Figur, deren ideologisiertes Bewußtsein Biographie sprachlich nur als Schema zuläßt. «Bis tief in die Arbeiterklasse hinein hatte die antisowjetische Hetze ihr Werk getan. Diese Menschen zu erziehen war eine gigantische Aufgabe» (Ma 202). Die Schuld, die dieses Opfer des Faschismus als Täter in der DDR auf sich geladen hat, hält ihm die Ich-Erzählerin in einem Augenblick vor, der seinen Tod bedeutet. Begleitet wird das Geschehen von ihren eigenen Mordphantasien. Das Opfer-TäterSyndrom scheint sich zu wiederholen. «Es bringt mich um, daß ich so froh sein kann, wenn andere sterben» (209). Diese Selbsterkenntnis der Ich-Erzählerin, nunmehr nicht mehr als alternative, sondern als in sich widersprüchliche Moral, ist doch zu melodramatisch inszeniert, um Müllers Hinweis überzeugend gerecht $\mathrm{zu}$ werden (Auch wenn der Text das selbst vielleicht reflektiert: Die «Hände» der Erzählerin, die sich nur imaginativ um Beerenbaums Hals gelegt hatten, üben am Ende wieder «Tonleitern», 209, 218). Dabei ist der Roman strukturell stimmig angelegt. Das dogmatisierte teleologische Erzählmuster Beerenbaums wird nur zitiert, während das Ich der Erzählerin es im achronologischen Arrangement der Erzählsequenzen gerade in Frage stellt: Beerenbaums Beerdigung als eigentliches «Telos» steht am Anfang des noch in der DDR angesiedelten Romans und wird im Verlauf der Handlung als Gegenwartsebene beständig in Erinnerung gerufen. Jedenfalls unterminiert diese «Gesinnungsästhetik» moralische Glattstellungen sozusagen mit Gewalt, wenn sie auch kaum überzeugend plausibel machen kann, daß es nicht an der Zeit ist, Moral und Fiktion (Ästhetik) zu trennen.

10 Jurek Beckers letzter Roman Amanda herzlos $(1992)^{15}$ schließt sich erzähltechnisch und thematisch gesehen hier an, macht aber den ganz gewöhnlichen DDR-Alltag anschaulich, in dem nicht das moralisch aufgeregte Ich die Realitätswahrnehmung (und den Leserzugang) prägte, wie bei Maron oder Christa Wolf, sondern das mehr oder weniger deformierte Ich, das sich im apparativen Gestänge sein durchaus passables 
Leben zurechtmachte. Auch die grotesken Züge daran blieben immer Lebensalltag. Mimesis traditioneller Art ist da für Becker angesagt, nicht spielerisch-satirische Phantastik wie etwa bei Irmtraud Morgner. Natürlich hat Beckers mimetisches Verfahren jede Naivität verloren. Wo Kant mit dem Abbruch seiner autobiographischen Teleologie zu kämpfen hatte, die bei Maron als dogmatische Selbstformierung nur noch zitiert wird, da wird derartiges in Beckers Roman schon in den Überschriften der drei Romanteile ironisch konterkariert: «Die Sheidung» - «Die verlorene Geschichte» - «Der Antrag», lies: der Heiratsantrag. Also eine Kreisbewegung um den Verlust herum, die nur, wenn «Der Antrag» weiterführte ${ }^{16}$, ein progressives Ziel anvisieren könnte. Teile von Lebensgeschichten erzählt der Roman immerhin, und am Ende winkt, Anfang 1989, eine Übersiedlung in den Westen. «Wir geben uns ein halbes Jahr, und wenn du danach zurück willst, dann ziehen wir wieder zurück» (Ah 384). Die inszenierte Distanz zu den Veränderungen der letzten Jahre gehört zu den Kunstmitteln des Romans, die ihn von der dramatisierten Nähe der Ich-Erzählerin bei Maron unterscheiden.

11 Schon der Titel, so betont trivial er wirkt, reizt zum Kommentar. Daß es sich um eine Art Oxymoron handelt, ist schon als Motto meiner Ausführungen gedeutet worden. Aber ebenso möglich ist die Lesart des Namens als Objekt einer elliptischen Verbalphrase, passivisch formuliert: «Amanda (wird) herzlos gesehen». Oder noch schlimmer: Man hat Amanda das Herz geraubt, nun ist sie herzlos geworden. Die Trivialität des Romantitels bleibt trotz solcher semantischen Konnotationen unangetastet. Und das ist auch gut so, denn der Roman berichtet ja nichts Sensationelles, sondern pointiert das Übliche, erzählt gegen den Strom der lauten öffentlichen Debatten. «Ich glaube, ich verlange nichts Unmögliches. Scheidungen sind nun mal ihrem Wesen nach unangenehm [...]» (Ah 9). So beginnt der Text, und er schließt im gleichen Tonfall, nur mit einer kalauernden Anspielung auf eine repräsentative Frucht der Wendezeit: «Und weißt du, daß es an jeder Ecke Bananen zu kaufen gibt? So ein Unglück ist das ja auch nicht» (384).

Beckers Titel verheißt eine weibliche Hauptfigur, deren Name mit Irmtraud Morgners Amanda. Ein Hexenroman (1983) schon einmal einen Titel lieferte. Morgners phantastischer Montage-Roman war ein Beitrag zur «Frauenliteratur», zweiter Teil einer unvollendeten Trilogie, deren erster Band Leben der Trobadora Beatriz 1973 die Frauenliteratur in der DDR mit begründet hatte. Morgners Amanda ist ein ungeniert fabulierender Roman, der für die «ketzerischen Potenzen $»^{17}$ der Frauen wirbt, deren «Schloß Blocksberg» unzweifelhaft in einem militärischen Sperrgebiet der DDR liegt. Amanda erscheint als die verschüttete Hälfte der S-Bahn-Führerin Laura Salman, von der die neuerlich auferstandene Trobadora Beatriz erzählt. Beckers Amanda wird dagegen nicht von einer sympathisierenden Trobadora freiphantasiert, sondern von drei ziemlich alltäglichen männlichen Ich-Erzählern verdeckt. Das Bedeutungsspiel des Titels erhält seine strukturelle Begründung: In drei chronologisch locker verknüpften Anläufen, den drei Roman-Teilen, erscheint Amanda in der Perspektive des männlichen Blicks, herzlos, herzlos gesehen, ihres Herzens beraubt, offenbar niemals «erkannt», in jedem Fall sprachlos (es sei denn in referierten Dialogen). Zwei der Sprecher sind Männer aus der DDR (Ludwig Weniger, ein linientreuer Journalist, Fritz Hetmann, ein regimekritischer Schriftsteller); einer kommt aus dem Westen (Stanislaus Doli, der Rundfunkkorrespondent), er plant die schließliche Übersiedlung mit Amanda dorthin. Amanda bleibt «Objekt»; sie wird tendenziell niemals «Ich», ist immer «sie». Da mittels der chronologischen Addition der drei Erzählungen von ihr aber erzählzeitlich am längsten und perspektivenreichsten berichtet wird, wirkt Amanda schemenhaft, weil 
sie immer von außen gesehen ist, und widersprüchlich zugleich. Man muß sich nicht sofort auf das schon topische Deutungsmuster einlassen, Amanda erscheine als das «Andere» der männlichen Erzählerstimmen, obwohl das nicht falsch wäre. Wichtiger ist zunächst die Beobachtung, daß Amanda zunehmend von den Ordnungen abrückt, die von den männlichen Sprechern des (kulturellen) Establishments repräsentiert werden, hinter denen wiederum die (kultur-) politischen Apparate erkennbar werden. Die männlichen Ich-Erzähler versuchen ihre Biographie diskursiv zu stabilisieren, indem sie Amanda erzählend, erklärend, sich entschuldigend vereinnahmen oder abstoßen, während Amanda zunehmend unberechenbarer wird, soweit sich das im berichtenden Zugriff auf sie erkennen läßt. «Was immer in ihrer Ehe vorgefallen ist, es liegt viele Jahre zurück, Amandas Wut aber ist frisch, beinahe entstellend [...] Woher kommt es, daß ich jedes Wort Amandas kritisch bewerte?» (Ah 359) «Amandas Ja klingt ein wenig gepreßt, zumindest bilde ich mir das ein, nicht so vorbehaltlos und freudig wie meines» (376). So Stanislaus, der Westdeutsche, der Tagebuch führt, während seine DDR-Kollegen Monologe an den (impliziten) Leser richten, wobei sich die Haltung der drei nicht prinzipiell unterscheidet. Stanislaus schwant zwar, daß «die zu Liebende», Amanda, sich ihm entzieht, «daß der eine Liebesfall anders liegen könnte als der andere» (299 f.), aber er kümmert sich vor allem um die bürokratischen Maßnahmen der Übersiedlung in den Westen.

So plausibel Amanda herzlos sich im Kontext der autobiographisch-biographischen Rückblicke auf die DDR verstehen läßt, so wenig ist die Form des Romans nur aus diesem Kontext zu erklären. Beckers Romane (mit Ausnahme von Schlaflose Tage) bieten eine Vielzahl von Varianten der Icherzählung an. Da gibt es den peripheren IchErzähler in Jakob der Lügner (1969) oder im Boxer (1976), den verhältnismäßig distanzlosen Ich-Erzähler in Irreführung der Behörden (1973), der sich schließlich durch seine Anpassung an die realsozialistische Kulturpolitik selbst irreführt, und den Sohn, dessen Erzählung vom Vater zumindest so wichtig ist wie das, was er von sich selbst erzählt, in Bronsteins Kinder (1987). Amanda herzlos schließt strukturell am ehesten hier an, natürlich mit ganz anderem Personal und völlig veränderter thematischer Gewichtung. Die Spannung zwischen peripherem Ich-Erzähler, erzählter «Geschichte» und der darin erscheinenden Figur, um die es eigentlich geht (wie offenbar auch in Amanda herzlos), wird am nachdrücklichsten zu Beginn des Romans Der Boxer zur Sprache gebracht. Im Gegensatz zu Amanda kann Aron/Arno Blank gegen das Verfahren seines «Interviewers» protestieren: «Ich hätte mir vorgenommen, ihn zu verfeinern, ihn druckreif zu machen, ihn als Rohmaterial zu benutzen. Ich hätte von Anfang an meine eigenen Vorstellungen von einer Geschichte gehabt». Das ganze Vorhaben sei gescheitert, weil «wir immerzu deuteten». Im Erzählen sei ihm, Arno Blank, «oft die Frage gekommen [...]: das soll er gewesen sein? [...] ja, er war es, wer sonst, es war ja kein anderer da» ${ }^{18}$. Das könnte auch Amanda sagen, wenn sie auf Wort und Schrift ihrer Erzähler direkt reagieren dürfte. Die beiden Romane, deren IchErzähler selbst Hauptpersonen des Erzählten sind, gehen nicht den Weg der zerfasernden Selbstanalyse, den wir aus der Erzählprosa Christa Wolfs kennen. Vielmehr sind nun die Ich-Erzähler in Gefahr, ihrer eigenen Geschichte und darin den anderen Figuren nicht gerecht zu werden, sei es, daß sie die Selbstreflexion meiden oder daß sie erzählte Figuren eben nur auf ihre Weise verstehen. Alle Erzähler/ IchErzähler Beckers sind also Varianten eines Grundtypus, die der Wahrheit im faktischen, historischen und moralischen Sinn zwar nachspüren, um aus ihr eine stimmige «Geschichte» zu machen, die damit die Wahrheit aber auch verfehlen 
können. Es sind, anders gesagt, Varianten von Jakob dem Lügner, der ja noch das Beste wollte, während sich andere Erzähler, wie die drei Männer in Amanda herzlos, nur ihre Lebenslüge auf Kosten Amandas zurecht machen, die selbst kaum zu Wort kommt. Die Wahrheit ist (und bleibt) zu ihrem selbstbezogenen Sprechen peripher. «Du hast doch keine Ahnung von mir, sagt Amanda» (302).

Die drei Ich-Erzähler, die längeren Abschnitten ihres Lebenslaufs Sinn zu geben versuchen, indem sie von ihrem Verhältnis zu Amanda erzählen, sind alles andere als Identifikationsfiguren für den Leser. Ebensowenig sind sie freilich a priori satirisch verzerrt - von einem Autor, der vorweg schon alles besser wüßte. Wie die Literaturkritik bemängelt hat ${ }^{19}$, ist ihr Erzählen stilistisch als eine Art mündlichen Palavers angelegt. Man plaudert so hin; und sicher könnte da manches kürzer gefaßt sein. Das fingiert Selbstverständliche des Sprechens und der Ereignisse hat indes nichts vom Versöhnlichen eines Strittmatter.

Also: Den Wagen möchte ich behalten. Wir haben ihn mit Hilfe der Redaktion bekommen, mit Hilfe meiner Redaktion [...] In meinem Beruf ist man ohne Auto halbtot, Amanda dagegen braucht keinen. Außerdem fährt sie so, daß es einem den Magen umdreht. Wenn ich ihr Feind wäre, würde ich sagen: Nimm das Auto und fahr los (Ah 9).

Die Teilung des Besitzes vor einer Scheidung (Erzählrahmen des ersten Teils von Amanda herzlos): das Ich stärkt sich in seinen bürgerlichen Ansprüchen (mein Beruf, meine Redaktion), seinen Fähigkeiten und vor allem seinem Haben und Behalten. Amanda werden Bedürfnisse und Fähigkeiten abgesprochen. Das Schlimmste, die Kriegserklärung («wenn ich ihr Feind wäre»), wird gerade noch verhindert. Indem sich die Rollen stabilisieren, entsteht ein peripheres Vakuum der Ich-Erzählung, das von dem Namen «Amanda» herausfordernd besetzt ist. Solches Erzählen zeigt, was Horváth «Demaskierung des Bewußtseins» genannt hat ${ }^{20}$. «Es könnte sein, daß ich mich heute verliebt habe, ich werde der Sache nachgehen» (Ah 259). So beginnt der dritte Teil des Romans in Tagebuchform. Die Horváth-Anspielung ist vielleicht kein Zufall, jedenfalls leitet der namenlose Ich-Erzähler in Horváths Kurzgeschichte «Die gerettete Familie» seine Geständnisse mit dem Satz ein: «Am 7. August 1922 war ich sehr verliebt und zwar in eine gewisse Frau Tomaschek aus dem VIII. Bezirk» ${ }^{21}$. Bei Beckers Stanislaus handelt es sich um den «Sept. 87», und die Verliebtheit hält länger an. Horváths Einsichten in die Selbstentlarvung der Sprechenden ließen sich zwanglos auf Beckers Ich-Erzähler übertragen, obwohl die sich einer fingierten Mimesis verdanken, die das Palaver weniger verfremdet als Horváth den kleinbürgerlichen «Bildungsjargon». Doch macht Beckers Erzählverfahren das Bemühen um Identität als fragwürdige Selbstbehauptung gegenüber einem von vornherein Nicht-Identischen durchschaubar, eben Amanda. Stanislaus erhofft sich folgerichtig von ihr den «Lohn» für sein langjähriges Ausharren «auf dem Vorposten der Freiheit» (Ah 259).

Drei mutmaßende Erzählungen über Amanda also. Das erinnert, wiederum kaum zufällig, an das bedeutendste Vorbild, Johnsons Das dritte Buch über Achim, das seinerzeit aus westlicher Perspektive die Unmöglichkeit zeigte, der DDR-Realität hinter ihrer ideologischen Heldenfassade auf den Grund zu kommen. Becker hat die Ausgangslage vereinfacht und verschoben: Ein Mitläufer (Ludwig) und ein Opponent (Fritz) aus der DDR erzählen demaskierend von sich, da bleibt am DDR-Alltag nichts rätselhaft. Der westdeutsche Journalist Stanislaus hat zwar erhebliche Schwierigkeiten, als er mit seiner Frau Amanda ausreisen will, aber auch für ihn spielen sich alle Absurditäten der DDR-Realität im Bereich des irgendwie Kalkulierbaren ab, ganz anders als für Johnsons 
Karst. Das Banale des Alltags und das mehr oder weniger Beiläufige der staatlichen Kontrollmechanismen bilden die Grundlage des Geschehens. Auch mit der Stasi und anderen Abteilungen des Staatsapparats hat man auf die eine oder andere Weise einen modus vivendi gefunden. "Rudolf gab sich Mühe herauszufinden, wer von den Anwesenden zum Staatssicherheitsdienst gehörte, aber er wollte sich nicht festlegen» (Ah 121), so Fritz in seiner autobiographischen Novelle. Die detaillierte Beschreibung, die auch bei einer «Lesung [...] pamphletistischer Art» in einer Privatwohnung nicht ausläßt, daß ein «Stuhl klebte» (120), das realistische understatement sind ein Grundduktus aller Erzähler. Das unauflösliche Ineinander von im Grunde völlig normalen, auf DDR-spezifische Weise geregelten Lebensverhältnissen und der dauernden Kontrolle durch die Apparate, mit denen man umzugehen lernt, um seine eigenen Interessen zu verfolgen, so gut es geht, ist zur Realität auch des westdeutschen Beobachters geworden. Ende 1987 muß sich dieser akklimatisierte Auslandskorrespondent erst von einem derangierten Alkoholiker-Kollegen auf das Kommende hinweisen lassen:

Ich habe nicht viel von Unruhe gemerkt [...]. Er spürt, daß etwas heranwächst. [...] Die Leute seien bisher ohne Verstand unglücklich gewesen, nun würden sie sich langsam ihres Unglücks bewußt, das sei der entscheidende Punkt (276).

Die Kontrastfolie dieser Beschreibung karikiert Anfang 1988 (Romanzeit) ein Anwalt, mit dessen Hilfe Stanislaus die Übersiedlung Amandas in den Westen bewerkstelligen will:

Glauben Sie mir, sagt er, diese Leute [...] sind es gewohnt, in Gehegen zu existieren, alles Unerwartete versetzt sie in Panik. Sie haben etwas Kuhiges, sie malmen ihr Gras, glotzen den Horizont an und wollen pünktlich gemolken werden (299).

Damit soll nicht einer primär politischen Lesart des Romans das Wort geredet werden, denn schon der Titel signalisiert ja anderes. Die alte Spannung zwischen «Privatem» und öffentlicher Rolle gehört zur thematischen Konstruktion. Ohne Zweifel geht es auch um die Gegenbewegung zwischen den Interessen und fragwürdigen Identitäten der drei Ich-Erzähler und der Identitätssuche Amandas. «Das Maß bin ich» (18), referiert Ludwig von ihr, und Fritz zufolge «habe sie die Bemühung um eine auffälligere Biographie nicht aufgegeben» (174). «Weiblichkeit ist nicht eine biologische oder kulturelle Identität, sondern das differentielle Moment, das Identität erst ermöglicht, in der zustande gekommenen Identität aber verdrängt wird $»^{22}$. Diese These aus der dekonstruktiv-feministischen Diskussion ließe sich durchaus auf den Roman übertragen, freilich mit Einschränkungen: Die (höchst fragwürdige) Alternative, das «nicht» - «sondern» zwischen biologischer und kultureller «Identität» und «differentiellem Moment» bringt der Roman entschieden ins Wanken, weil er auf kulturellen und biologischen Bedingungen beharrt, ja die erzählte Amanda vielleicht mehr als einträglich mit solchen Momenten besetzt ${ }^{23}$. Während Amandas Kollision mit der Stasi im ersten Teil des Romans eher auf einem Mißverständnis beruht, hat sie im zweiten und vor allem im dritten Teil offenbar an politischem Bewußtsein gewonnen und Kontakt zu oppositionellen Gruppen etwa der Friedensbewegung aufgenommen. Und je weniger sie von ihren Männern erwarten kann, desto mehr hängt sie an ihrem Sohn; ein Gespräch mit ihm referiert Stanislaus als Romanschluß. Zugleich werden die männlichen Identitäten nicht als, wie Vinken sagt, «zustande gekommene», sondern als zweifelhafte Bemühungen um Identität, immer in Beziehung zu der «abwesenden» Amanda, erzählt. 
19 Alle vier Hauptpersonen, die drei anwesenden und die abwesende, sind beruflich mit Texten beschäftigt. Ludwig, der DDR-Zeitungsredakteur, Stanislaus, der westdeutsche Rundfunkjournalist in der DDR, haben es mit Gebrauchstexten zu tun. Der literaturpolitisch unterdrückte DDR-Autor Fritz Hetmann ist als einziger mit belletristischen Texten befaßt. Seine Erzählung ist folgerichtig komplexer angelegt als die der beiden Kollegen. Ihm ist eine Novelle auf dem Computer verlorengegangen, die er nun bruchstückhaft rekonstruiert, weil sich darin sein vergangenes Leben mit Amanda spiegelt. Während er also sein Privatleben zur Literatur verarbeitet, die vielleicht eines Tages das Licht der literarischen Öffentlichkeit im Westen erreichen wird, da Hetmann in der DDR nicht mehr gedruckt werden darf, repräsentieren die IchErzählungen der beiden Journalisten die Spannung zwischen (erotischem) Privatleben und politischer Öffentlichkeit. Ludwig steht dabei für die DDR intern, Stanislaus für den westlichen Blick auf diese DDR. Damit ist die hochgradig selbstreflexive Erzählstruktur des Romans, der seinerseits 1992 Bestandteil der gesamtdeutschen literarischen Öffentlichkeit geworden ist, aber noch nicht vollständig beschrieben. Die drei Männer verkörpern auch Varianten öffentlichen Sprechens, zu denen Amanda in Opposition gerät.

In der Ehe mit Ludwig hat sie zum Unbehagen ihres Mannes, anders als in der DDR üblich, keine feste Stelle, sondern nimmt nur freie Aufträge als Reporterin an. Dies Engagement vermindert sich zusehends, weil sie an einem Roman schreibt, von dem wir nur einige Details erfahren, nachdem sich Ludwig Einblick in das Manuskript verschafft hat. «Die Geschichte, von der sie schrieb, handelte von einer Kindheit, ich nehme an, von ihrer eigenen. Ich las natürlich nur Ausschnitte» (AH 77). Es geht um kindlich-widersetzlichen Alltag mit Mutter und Vater, offenbar erzählt aus der Sicht des kleinen Mädchens, soweit Ludwigs Referat das erkennen läßt. Eben diese Art der Selbsterkundung ohne auktoriale Einrede und die «tödliche Ausführlichkeit» (79) in der Darstellung des Alltäglichen müssen ihm zuwider sein, der - das braucht nicht eigens gesagt zu werden - an soz-realistisch «Typisches» gewöhnt sein wird. «Vielleicht wird sie eines Tages eine Berühmtheit, allerdings würde mich das wundern. Für viel wahrscheinlicher halte ich, daß die Sache im Sande verläuft» (32). So Ludwig schon zu Anfang, und am Ende des Romans zeigt sich, daß er fast recht behält. Der Roman im Roman wird jedenfalls nie fertig.

Dennoch spielt er auch bei Fritz, dem zweiten Ich-Erzähler, noch eine Rolle, erwartungsgemäß eine negative. Die «Gefahr, die uns daraus drohte» (162), ist nicht politischer, sondern offenbar rein literarischer Art. Fritz bemängelt den sprachlichen Stil Amandas und - darin trifft er sich mit Ludwig -, «daß sie offenbar glaubte, der Banalität vieler beschriebener Vorgänge mit Ausführlichkeit beikommen zu können, daß ihr Erzählzug [...] keinen Zielort hatte» (162). Anders offenbar als seine eigenen Erzählbände, dessen jüngster endlich in der DDR verboten wird, und den er dann ungenehmigt im Westen herausbringt. Fritz hat ein Gespräch mit Amanda über seinen eigenen neuen Roman (als Debatte zwischen Rudolf und Louise) in seine verlorene Novelle eingebaut: «Anstatt einfach zu sagen, es sei sterbenslangweilig gewesen, sage er: Langweilig wie auf einer Parteiversammlung. Sticheleien seien dem Autor wichtiger gewesen, als etwa zu beschreiben, wie Johannes sich nach dem Aufstehen die Zähne putzte» (214). Louise-Amanda verlangt von Rudolf-Fritz eben das, was er ihr als Mangel vorwirft: die Beschreibung des Normalen, aus dem vielleicht das Symptomatische hervorgeht, während die verbrauchte auktoriale Stichelei nur den Lektor-Zensor 
irritiert. Daß es ihr durchaus um kritischen Realismus zu tun ist, zeigt sich später, in Stanislaus' TagebuchErzählung. Er hält Amanda vor, er habe sich als Außenstehender «nicht um die Aufdeckung von Mängeln und um deren Beseitigung zu bemühen, sondern um Beschreibung. Amanda sagt: Schade» (269). Diese scheinbaren Widersprüche Amandas repräsentieren nicht einfach die «immanente Poetik» von Beckers Roman, aber sie stehen ihr nahe.

Amanda scheint ihren Roman allerdings (zeitweise) aufzugeben. Während sie gegen Ludwig mit freien Mitarbeiter-Verträgen und einem Romanprojekt opponierte, das sie in Verdacht brachte, verbotene Westkontakte aufzunehmen, während sie Fritz mit einem Beschreibungsroman konfrontierte, nimmt sie bei Stanislaus die offen oppositionelle Tätigkeit ernster, die sie schon am Ende der sieben Fritz-Jahre begonnen hatte. «Man rede über die täglichen Ungeheuerlichkeiten, für die man schon den Blick verloren habe, man diskutiere Sinn oder Unsinn von Widerstand, man übe sich darin, vielen Dingen wieder ihren richtigen Namen zu geben; und das alles nicht zu Hause [...], sondern öffentlich» (228). Und später: «Nach Jahren des Stillhaltens habe sie sich nun zu einer minimalen Tat entschlossen» (248). Amandas Realismus-Verlangen, offenbar begonnen mit einem fragmentarischen autobiographischen Roman über ihre Kindheit, bekommt schließlich eine pragmatische Funktion, die sich in der Zeit mit Stanislaus verstärkt, um dann im Plan des Westumzugs zu versanden. Dem RundfunkKorrespondenten Stanislaus, der selbst ahnt, «kein glänzender Journalist zu sein» (313), erwächst in Amanda eine publizistische Konkurrenz, die ihre schärfste Kritik nur über den Westsender öffentlich machen könnte. Natürlich nicht im eigenen, sondern in Stanislaus' Namen. «Ich muß ihr das Theater schildern, das es gäbe, wenn ich meine Sendezeit für Briefe und Manifeste aus dem Untergrund zur Verfügung stellte. [...] Während ich rede, knöpfe ich ihre Bluse auf, sie scheint es nicht zu bemerken» (293). Stanislaus muß für seine Rundfunkberichte Rügen einstecken, weil er die redaktionelle Kontrolle versäumt hatte; den «monierten Text habe ich nicht geschrieben, Amanda war es» (353). Am Ende schließt sich der Ring der männlichen Erzählungen, der Amandas letztlich aussichtslose Artikulationsversuche einhüllt. Nicht nur der Weg zur «Tat», schon der Weg zum Wort im eigenen Namen ist ihr versperrt. So nimmt sie ihren Roman wieder auf. Für Stanislaus ist es «merkwürdig [...], plötzlich mit einer Schriftstellerin verheiratet zu sein», wie zuvor für Ludwig und Fritz. «Ob ich sie dann nicht geheiratet hätte. Ich sage, das hängt von deinem Roman ab, zeig her das Ding. Doch sie schüttelt den Kopf und möchte in Ruhe gelassen werden» (380).

Die achtziger Jahre. Ein Jahrzehnt der laufenden Ereignisse, das durch die drei IchErzähler repräsentiert wird, hat an den Strukturen nichts geändert: Erzähler, die Amandas Provokationen in Bestätigungen ihrer eingeschliffenen Lebensformen umbiegen, und sei es durch Scheidung, Trennung oder Heirat. Gleichviel. Auf der anderen Seite Amandas keineswegs heroische, sondern eher hilflose Versuche, aus der peripheren Existenz zu einer privaten und öffentlichen Artikulation des Ich zu finden. Was ihr am Schluß bleibt, ist das melancholische Gespräch mit dem kleinen Sohn - und der unvollendete Roman, der das Ende an den Anfang knüpft, nun allerdings unter «Bananen»-Verhältnissen.

24 Apologetik und Literarisierung des autobiographischen Ich (Kant, Müller), Gleichwertigkeit (Strittmatter), moralische Dramatisierung des Opfer-Täter-Syndroms durch die aufgeregte IchErzählerin (Maron) - Amanda herzlos scheint all dies zu reflektieren. Moral und Literatur sind da nicht geschieden, aber Beckers Roman ist 
zugleich weit entfernt vom moralisierenden Gestus. Was er dem literarischen Lesepublikum im vereinten Deutschland $\mathrm{zu}$ bedenken gibt, sind fingierte IchErzählungen, in denen es den Angepaßten nicht schlecht geht, denen sich aber Amanda entzieht. "Alle meine Stücke sind Tragödien - sie werden nur komisch, weil sie unheimlich sind. Das Unheimliche muß da sein». So Horvath ${ }^{24}$. Im Unheimlichen ist die Wahrheit verdrängt, und das ist spürbar. Worin die Wahrheit besteht, wissen wir auch in Beckers Roman nicht, weil Amanda nicht zu Wort und schon gar nicht zur «Tat» kommt.

\section{NOTES}

1. - Wichtige publizistische Beiträge dazu sind gesammelt in: Thomas Anz (Hrsg.), «Es geht nicht um Christa Wolf». Der Literaturstreit im vereinten Deutschland, München, 1991. - Vgl. außerdem Karl Deiritz/Hannes Krauss (Hrsg.), Der deutsch-deutsche Literaturstreit oder: Freunde, es spricht sich schlecht mit gebundener Zunge. Analysen und Materialien, Frankfurt/M, 1991.

2. - Vgl. dazu eine der neuesten Arbeiten: Oliver Sill: Zerbrochene Spiegel. Studien zur Thematik und Praxis modernen autobiographischen Erzählens, Berlin-New York, 1991.

3. - Ulrich Greiner, «Die deutsche Gesinnungsästhetik», in Th. Anz (Hrsg), Literaturstreit, S. 208-216; hier S. 216.

4. - Vgl. u.a.: Thomas Rietzschel, «Mit einer gewissen intellektuellen Ängstlichkeit. Beschreibung einer Akte: Wie Christa Wolf als IM für die Stasi gearbeitet hat», in FAZ, Nr. 18. 22.01.93; «Die ängstliche Margarete», in Der Spiegel A (1993). S. 158-165.

5. - Vgl. u.a.: Heiner Müller, «Ein Erklärung und neue Dokumente», in FAZ, Nr. 13. 16.01.93; Frank Schirrmacher, «Fälle. Wolf und Müller», in FAZ, Nr. 18. 22.01.93; Iris Radisch, «Krieg der Köpfe», in Die Zeit, Nr. 4. 22.01.93; Fritz J. Raddatz, «Von der Beschädigung der Literatur durch ihre Urheber. Bemerkungen zu Heiner Müller und Christa Wolf», in Die Zeit, Nr. 5. 29.01.93.

6. - «Vermisse das Wort Pinscher». Ein Staatsschriftsteller im Stasi-Dienst: Die Spitzel-Karriere des Genossen Hermann Kant alias IM «Martin», in Der Spiegel 41 (1992). S. 323-336; Hermann Kant, «Ich, der Geheime. Stellungnahme zu den Stasi-Vorwurf en», in FAZ, Nr. 232. 6.10.92. Dazu, «Alles abstreiten. Günter Kunert und Wolf Biermann antworten Hermann Kant», in FAZ, Nr. 233. 7.10.92. 7. - «Ein Beitrag zum Umgang mit den Stasi-Akten», in FAZ, Nr. 41. 18.02.93.

8. - Dagegen ließen sich andere Texte ins Feld führen, die Adornos Diktum jedenfalls durch ihr Stasi-Thema bestätigen, z.B. Reiner Kunze, Deckname «Lyrik». Eine Dokumentation, Frankfurt/M., 1990; Erich Loest, Der Zorn des Schafes. Aus meinem Tagewerk, Künzelsau-Leipzig, 1990.

9. - Hermann Kant, Abspann. Erinnerung an meine Gegenwart, Berlin und Weimar, 1991 Zitierweise: K, Seitenzahl.

10. - Heiner Müller, Krieg ohne Schlacht. Leben in zwei Diktaturen, Köln, 1992 - Zitierweise: M, Seitenzahl.

11. - Erwin Strittmatter, Der Laden. Roman dritter Teil, Berlin und Weimar, 1992 - Zitierweise: S, Seitenzahl.

12. - Dazu: Im Osten läuft der Laden, in Der Spiegel 45 (1992). S. 272-279.

13. - Zu den Kontexten etwa: Paul Gerhard Klussmann/Heinrich Mohr (Hrsg.): Probleme deutscher Identität. Zeitgenössische Autobiographien. Identitätssuche und Zivilisationskritik, Bonn 1983 (Jahrb. z. Lit. in der DDR. Bd. 3). Darin Beiträge über Loest, Fühmann, Schleef, Hermlin; Horst Domdey/ 
Michael Rohrwasser: «Stalinismus und die Ausklammerung der Renegatenliteratur», in Text + Kritik 108 (1990). S. 68-75.

14. - Monika Maron, Stille Zeile sechs, Roman, Frankfurt/M., 1992 - Zitierweise: Ma, Seitenzahl.

15. - Jurek Becker, Amanda herzlos, Roman, Frankfurt/M., 1992 - Zitierweise: Ah, Seitenzahl.

16. - Vgl. die Lektürevorschläge von Irene Heidelberger-Leonhard: «Schreiben [...] nichts anderes als eine endlose Reihe von Zweifeln, die zugunsten eines Satzes endlich überwunden werden müssen». Fragen an Amanda. In: I. H.-L. (Hrsg.): Jurek Becker, Frankfurt/M., 1992 (suhrk.tb. materialien). S. 301-311.

17. - Irmtraud Morgner, Amanda. Ein Hexenroman, Darmstadt - Neuwied, 1984, S. 528.

18. - Jurek Becker, Der Boxer, Roman, Frankfurt/M., 1979. S.U.

19. - Marcel Reich-Ranicki, «Drei Idioten. Jurek Beckers Roman "Amanda herzlos"», in Die Zeit, Nr. 219. 19.9.92.

20. - Ödön von Horvath: Gebrauchsanweisung. In: Ö.v.H., Gesammelte Werke. Hrsg. von Traugott Krischke u. Dieter Hildebrandt. Frankfurt/M., 1972. Bd. 8: «Denn letzten Ende ist ja das Wesen der Synthese aus Ernst und Ironie die Demaskierung des Bewußtseins. Sie erinnern sich vielleicht noch an einen Satz in meiner "Italienischen Nacht", der da lautet: "Sie sehen sich alle so fad gleich und werden gern so eingebildet selbstsicher". Das ist mein Dialog» (660).

21. - Ebd. Bd. 5. S. 87.

22. - Barbara Vinken, «Dekonstruktiver Feminismus. - Eine Einleitung», in B.V. (Hrsg.), Dekonstruktiver Feminismus. Literaturwissenschaft in Amerika, Frankfurt/M., 1992. S. 7-32; hier S. 19.

23. - Vgl. die analogen Thesen der Sozialpsychologie von Hans-Joachim Maaz, Die Entrüstung. Deutschland, Deutschland, Stasi, Schuld und Sündenbock, Berlin, 1992; ähnlich Christoph Dieckmann, «Abendlicht. Eine Predigt für und wider den Mythos DDR», in Die Zeit, Nr. 9. 26.02.93.

24. - Horvath, GW. Bd. 8. S. 664. - Beckers Roman ist sicher nur metaphorisch «unheimlich». Noch weniger läßt er sich einfach auf Müllers Literarisierung der Historie auslegen: «Im Drama seit Shakespeare steckte die Farce im Bauch der Tragödie, mit dem Bankrott der sozialistischen Alternative geht die Ära Shakespeares zu Ende, und im Bauch der Farce lauern die Tragödien» (M 344).

\section{RÉSUMÉS}

Im Zusammenhang mit der 1990/93 vor allem feuilletonistisch gefürhrten Debatte über die politische Orientierung, moralische Verantwortung und (Stasi-)Verstrickung von DDR-Autoren kommt der neuesten (quasi-autobiographischen Erzählprosa ein genuines Erkenntnispotential zu. Zwar werden in ihr referentielle Inhalte erzählt, aber zugleich reagieren (H. Kant) oder insistieren (H. Müller) die Texte darauf, daß das seit der Jahrhundertwende thematisierte «unrettbare Ich» (Bahr), daß Sinnverlust und Mutmaßungen über authentische Wahrheit nicht nur Schlagworte sind. Je augenfälliger die Fiktionalisierung jüngster Vergangenheit wird, desto überzeugender wird die Realitätsdeutung zum literarischen Entwurf, der diese Prämissen selbstreferentiell aufnimmt - tröstlich bei Strittmatter (Der Laden), in polemischer Opposition gegen falsche Teleologie bei Maron (Stille Zeile sechs). Jurek Beckers neuer Roman (1992) spitzt einerseits die für ihn bezeichnende Trennung des Erzählens vom Erzählten zu - insofern steht Amanda herzlos im Werkkontext-, andererseits macht der Roman die aktuelle Frage nach biographischer Kontinuität (oder lebensgeschichtlichen Brüchen), nach der erkenntnis der 
Wahrheit strukturell thematisch. Zwei DDR- und ein westdeutscher Intellektueller, auf unterschiedliche Weise mit Texten befaßt, versuchen ihre Biographie zu stabilisieren, indem sie sich erzählerisch, palavernd oder in literarischer Brechung des «Faktischen», einer Frau bemächtigen, die sich ihnen widersprüchlich schemenhaft entzieht, der Vereinnahmung ausweicht, selbst wenn ihr das am Ende, vor der Übersiedlung in den Westen (Januar 1989), nur in derselben Form möglich ist, wie am Anfang: indem sie ihr eigenes Romanprojekt weiter schreibt. Den Angepaßten (Männern) geht es gut, aber die Wahrheit über Amanda entzieht sich; Johnsons Mutmaßungen über erfahrbare DDR-Realität werden da implizit korrigiert, wo es nichts mehr zu mutmaßen gibt, aber sie werden nicht dementiert. Beckers Roman bleibt «moralische» Fiktion jenseits jeder «Gesinnungsästhetik» (Greiner).

$\mathrm{Au}$ vu du vaste débat mené dans les journaux littéraires en 1990-1993 sur les orientations politiques, la responsabilité morale et la compromission de certains auteurs de la RDA avec la Stasi, la prose narrative récente en partie autobiographique représente aujourd'hui une extraordinaire source d'informations. Cette prose véhicule sans doute un contenu référentiel, mais en même temps ces textes soit traduisent en réaction (H. Kant), soit affichent (H. Müller), que «l'irrémédiable dilution du moi » (Bahr) thématisée depuis le début du siècle, la perte du sens et les conjectures sur la vérité authentique ne sont pas que slogans. Plus la mise en fiction du passé immédiat est manifeste, plus convaincante est l'interprétation de la réalité en un projet qui fait de ses prémisses ses propres références, - que ce soit sur le mode de la consolation chez Strittmatter (Der Laden), ou d'une opposition polémique contre une fausse téléologie chez Maron (Stille Zeile sechs). Dans le nouveau roman de Jurek Becker (1992), la fracture entre la narration et le narré - si caractéristique de sa manière - atteint un point critique - Amanda herzlos s'inscrit bien dans cette mesure dans l'ensemble de l'œuvre - ; par ailleurs, la question actuelle de la continuité biographique (ou des ruptures existentielles), de la connaissance de la vérité, soustend toute la structure thématique du roman. Deux intellectuels de RDA, et un intellectuel d'Allemagne de l'Ouest, avec chacun sa relation particulière aux textes, tentent de donner un équilibre à leur biographie, en prenant possession d'une femme par le récit, la palabre, ou par la réfraction littéraire des "faits "; une femme qui se dérobe à eux de façon contradictoire et imprécise, échappe à la récupération, même si à la fin, avant de passer à l'ouest (1989) cela ne lui est posssible que sous la même forme qu'au début: en continuant d'écrire son propre roman. Ceux qui se sont adaptés (les hommes) vont bien, mais la vérité sur Amanda se dérobe; les conjectures de Johnson sur l'expérience possible de la réalité de la RDA se trouvent implicitement corrigées là où il n'y a plus rien à conjecturer, mais elles ne sont pas démenties pour autant. Le roman de Becker demeure de la fiction «morale " par-delà toute " esthétique de conviction » (Greiner).

\section{AUTEUR}

\section{LOTHAR KÖHN}

Westphälische-Wilhelms-Universität Münster 